\title{
A Single Sided Edge Marking Method for Detecting Pectoral Muscle in Digital Mammograms
}

\author{
Guliz Toz \\ Electrical, Electronic \& Computer Engineering Dpt \\ Duzce University \\ Institute of Science and Technology \\ Duzce, Turkey \\ glz.toz@gmail.com
}

\author{
Pakize Erdogmus \\ Computer Engineering Dpt \\ Engineering Faculty \\ Duzce University \\ Duzce,Turkey \\ pakizeerdogmus@duzce.edu.tr
}

\begin{abstract}
In the computer-assisted diagnosis of breast cancer, the removal of pectoral muscle from mammograms is very important. In this study, a new method, called Single-Sided Edge Marking (SSEM) technique, is proposed for the identification of the pectoral muscle border from mammograms. 60 mammograms from the INbreast database were used to test the proposed method. The results obtained were compared for False Positive Rate, False Negative Rate, and Sensitivity using the ground truth values pre-determined by radiologists for the same images. Accordingly, it has been shown that the proposed method can detect the pectoral muscle border with an average of $95.6 \%$ sensitivity.
\end{abstract}

Keywords-INbreast database; pectoral muscle extraction; segmentation; mammogram

\section{INTRODUCTION}

Mammography is one of the basic and effective imaging techniques used for the detection and diagnosis of breast cancer. Mammography is usually obtained in two forms, the mediolateral oblique view (MLO) and the craniocaudal (CC) view [1]. These two views are used to detect abnormal structures within the breast. However, because of the heterogeneity of the malignancies and overlapping of the intense fibro glandular tissue, it may be difficult to read and interpret mammograms in two-dimensional projection images [2]. Human factors as fatigue, limitation of the human eye and others may also cause misinterpretations. In addition, mammography alone is not sufficient for a final diagnosis and a biopsy may also be needed. However, a small percentage of breast biopsies are shown to be cancerous (only 15-30\% in case of [3]). Therefore, a variety of computer aided diagnostic systems are employed as a second reader to radiologists. Clinical trials indicate that the number of cancers detected by computer-aided diagnosis (CAD) systems is increased by about $10 \%$, which is comparable to double reading by two radiologists [4]. MLO-views mammograms often contain pectoral muscle. Since pectoral muscle shows similar features with abnormal structures such as mass, it is confused with suspicious regions in CAD studies and makes accurate diagnosis difficult [5]. For this reason, the removal of pectoral muscle from mammograms is important for accurate diagnosis.
The automatic removal of pectoral muscle from MLOviews mammograms is a necessary step. At the same time this procedure is quite difficult because values such as shape, size, and density in mammograms differ from mammogram to mammogram. The studies for detection of pectoral muscle from digital mammograms can be categorized in four main groups. These are density-based approaches $[6,7]$, line-based detection approaches [8,9], wavelet-based segmentation [10, 11] and statistical $[10,12]$ methods. Authors in [7] proposed a new adaptive method for the detection of pectoral muscle. In this method, the pectoral margin, position and orientation are estimated first with a suitable straight line. This line is then smoothed using the repeated "cliff detection" algorithm to draw the pectoral boundary more accurately. In [13], authors proposed an approach for the detection of segmentation at the pectoral muscle boundary based on the structure tensor. Experimental results indicate that the proposed method distinguishes the pectoral muscle exactly with the segments [13]. In [14], authors conducted a study based on the positional characteristics of the pectoral muscle. They have combined iterative Otsu thresholding and mathematical morphological processing to find the rough edge of the pectoral muscle. They applied multiple regression analysis to this rough border to obtain the correct segmentation of the pectoral muscle. In [15], authors combined median filtering, morphological erosion process, sobel edge detector and thresholding to find the breast rough limit, then use the GVF Snake algorithm with gradient map setting to obtain the sensitivity breast border. Authors in [16] used thresholding to identify pectoral muscles, connected component labeling to identify and remove the connected pixels outside the breast region and edge detection processes to identify the edge of the full breast. They used global thresholding for segmentation and removal of pectoral muscle. As a result, they noted that they have effectively removed the gauss and impulse noise and in general, they achieved $90.06 \%$ accuracy.

In this study, the pectoral muscle border was first determined by a new method called by the authors as Single Sided Edge Marking (SSEM), based on the geometrical properties of the pectoral muscle and neighborhood relations to extract the pectoral muscle from mammograms. Later, the 
points found were reinforced with morphological operators and a rough pectoral muscle region was obtained. The boundary of the found candidate pectoral muscle area has been reconstructed and this boundary extracted by the linear interpolation method has been redefined so that the missing point does not exist. The results obtained by the radiology specialists with the ground-truth data for the same images were compared in terms of false positive rate, false negative rate and sensitivity. Accordingly, it has been shown that the proposed method can detect the pectoral muscle border with a sensitivity of 95.6 percent.

\section{MATERIAL}

In this study, 60 mammogram received from the INbreast [17] database were used to test the methodology. The INbreast consists of 115 cases (410 images) of which 90 cases are from women with both breasts ( 4 images per case) and 25 cases are from mastectomy patients ( 2 images per case). Several types of lesions (masses, calcifications, asymmetries, and distortions) are included. Accurate contours made by specialists are also provided in XML format.

\section{PREPROCESSING}

Before performing pectoral muscle area boundary detection, noise reduction and image enhancement [18-22] on the image are necessary. The flowchart for all followed in the current work is presented in Figure 1. Firstly, the image has been reduced to a size of $512 \times 512$ and the pixel intensity values have been reduced to 256 for decrease processing complexity. All images are then arranged in such a way that the pectoral muscle region is in their upper left corner. In order to achieve this, a mirror image of the mammogram is taken if the pectoral muscle region is located at the upper right corner of the mammogram.



Fig. 1. Mammogram pre-processing flowchart

No additional procedure has been performed in the mammograms that have the pectoral muscle region in their upper left corner (Figure 2(b)). Then, the mammogram image has been converted to a binary image by using an obtained experimentally threshold value $\sigma=0.09$ (Figure 2(c)). Since, the largest region in the binary image is the breast region; a filter has been applied so that this area remains in the image. The resulting image was eroded using a two pixel structure element and then subtracted from the image obtained in the previous step. Thus, the rough border of the breast region has been obtained as seen in Figure 2(d). The resulting image has been multiplied by the original image and so the result is the image that contains only the breast region. Then, the $3 \times 3$ median filter and biorthogonal wavelet transform have been used to remove noise and enhance the image. Finally, the image has been enhanced with adaptive histogram equalization and the noise has been reduced by anisotropic diffusion method (Figure 2(e)).

The biorthogonal wavelet and anisotropic diffusion methods used for preprocessing are briefly described below.

\section{- Biorthogonal Wavelet Transform}

Wavelets are used in many areas including noise reduction in image processing. There are many wavelet families [23]. Biorthogonal wavelet representation can be seen to have many advantages when compared to orthogonal wavelet representation [24]. For example, sub-band images do not change and have no overlap under translation. Smoothing symmetric and asymmetric wavelet functions can be used to reduce the reflection of signal extensions and border effects [24]. Because of these advantages, a biorthogonal wavelet (biorthogonal 3.1) has been used for noise reduction in this work.

\section{- Anisotropic Diffusion Method}

Anisotropic diffusion method is a technique aiming at reducing image noise. This method reduces the image noise while preserving important image details, such as lines, edges, etc. that are important for interpretation of the image. The diffusion equation can be formulated as follows [14].

$$
\frac{\partial I}{\partial t}=\operatorname{div}(c(x, y, t) \nabla I)=\nabla c \cdot \nabla I+c(x, y, t) \Delta I
$$

where $c(x, y, t)$ is the diffusion coefficient. $c(x, y, t)$ is preserve edges in the image and usually chosen as a function of the image gradient which controls the rate of diffusion. The images related to the preprocessing operations are shown in Figures 2 (a)-(e).

\section{SINGLE SIDED EDGE MARKING (SSEM)}

\section{A. Pectoral Muscle Characteristics}

For the method proposed in this study, some peculiar characteristics of the pectoral muscle were utilized:

- The pectoral muscle region is a roughly triangular area.

- The pectoral muscle border is approximately a straight line.

- There is a certain density change between the pectoral muscle and the breast region.

- Pectoral muscle region is roughly homogeneous.

The SSEM method is based on the geometric characteristics of mammograms and the intensity difference between muscle and breast tissue. When we look at the geometrical properties of the mammograms, it is seen that it is a roughly triangular region that is narrowed from top to bottom. Since the pectoral muscle region is brought to the upper left corner in the preprocessing, the edge detection process is performed at an 
angle of about $30^{\circ}$ to $45^{\circ}$ from right to left and from top to bottom.

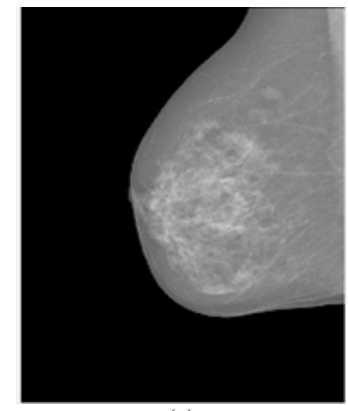

(a)



(c)

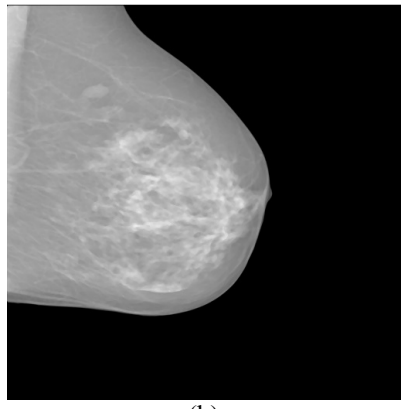

(b)

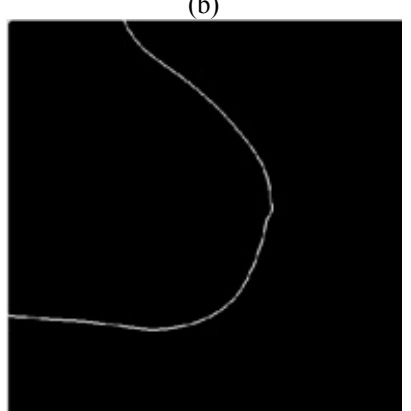

(d)

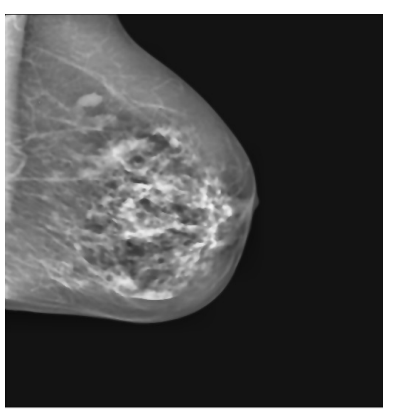

(e)

Fig. 2. (a) Original Mammogram. (b) Mammography mirror image, reduced to $512 \times 512$ dimensions and 256 pixel intensity values. (c) Segmented breast area. (d) Extracted breast rough limit. (e) Noise reduction and enhancement.

\section{B. SSEM Details}

First, three different threshold values have been defined for use in edge marking process. The first of these is the threshold value $(\varphi)$ used to exclude non-mammogram regions in the images; this value is taken as 5 . The second is the value $(\alpha)$ of how much the intensity values of the pixels are similar to each other and is taken as 1 in this study. The third threshold is the threshold value used for the density difference between the pectoral muscle and the breast region. This value is also set to 1 in this study. The fact that this value is kept small ensures that the pectoral muscle border is marked with as many points as possible. Next the mammogram image (I) scanned pixel by pixel, $I_{i, j}=(I=2,3, \ldots M ; j=2,3, \ldots, N-1)$, where, $M$ defines the number of rows of the matrix $\mathrm{I}$, and $\mathrm{N}$ is the number of columns. The selected pixel is evaluated together with its neighbors located at two pixel distances. The following conditions are used for this evaluation.

$$
\begin{gathered}
I(\mathrm{i}, \mathrm{j})>\phi \\
\text { and } \\
\left(\begin{array}{c}
\left.\mid \begin{array}{c}
\left|I_{i, j}-I_{i+1, j-1}\right| \leq \alpha \text { or }\left|I_{i, j}-I_{i-1, j+1}\right| \\
\text { or }\left|I_{i, j}-I_{i-1, j}\right| \leq \alpha \text { or }\left|I_{i, j}-I_{i, j-1}\right| \leq \alpha
\end{array}\right) \\
\text { and } \\
\left(\mid \begin{array}{c}
\left|I_{i, j}-I_{i, j+1}\right| \geq \varphi \text { and }\left|I_{i, j}-I_{i+1, j+1}\right| \geq \varphi \\
\text { and }\left|I_{i, j}-I_{i+1, j}\right| \geq \varphi
\end{array}\right) \\
I_{-} n e w_{i, j}=255 \text { and } \\
I_{-} n e w_{i+1, j-1}=255 \text { and } \\
\text { if }(j-2) \neq 0 \text { then } I_{-} \text {new } \\
\text { else } I_{i+2, j-2}=25 e w_{i, j}=I_{i, j}
\end{array}\right)
\end{gathered}
$$

As a result of the evaluation made according to these conditions, the new mammogram image marked I new is obtained on the border of the pectoral muscle. The reason for choosing pixel intensity values of 255 for marking the pectoral muscle margin, is that this is a value less common on the mammogram and easier to distinguish in binary images. A graphical representation of the SSEM method and a sample application are presented in Figure 3.



(a)

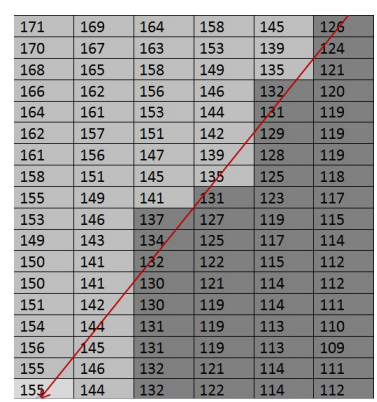

(b)

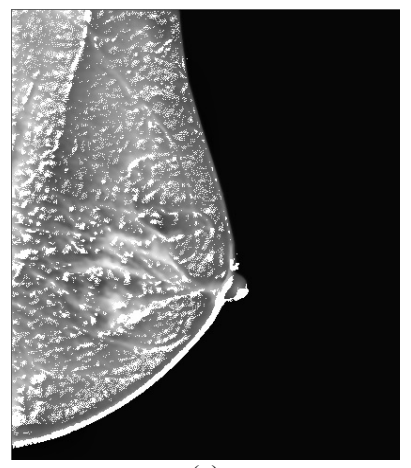

(c)

Fig. 3. Graphical representation of the SSEM method and a sample application (a) Graphical representation for border detection by SSEM method. (b) A sample pixel intensity values of 6x18 muscle and breast area. (c) New mammographic image with marked pectoral muscle border as the result of SSEM method. 


\section{Determination of Candidate Pectoral Muscle and Border Area}

In order to find the candidate pectoral muscle region from the image of the new mammogram marked with the pectoral muscle boundaries as the result of the SSEM method, two different variables, experimentally obtained and named as Area $_{\min }$ and Area $_{\max }$ were used in this study. Area $a_{\min }$ is the smallest possible pectoral muscle area and Area $_{\max }$ is half of the largest possible mammogram region. In Figure 4, there are sample mammogram images of Area $_{\max }$ and $\mathrm{Area}_{\min }$.

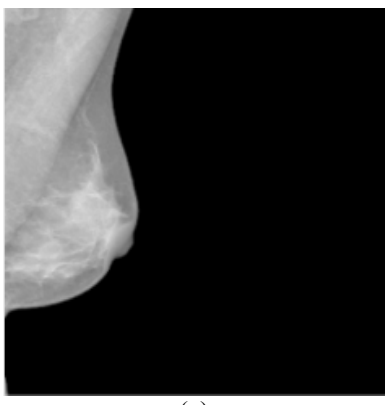

(a)



(c)

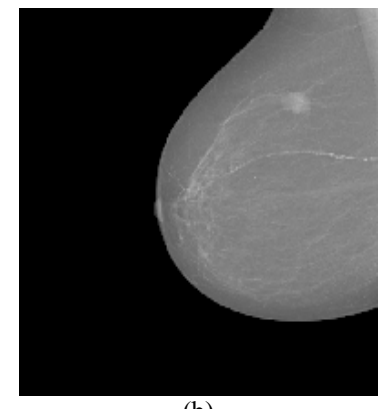

(b)

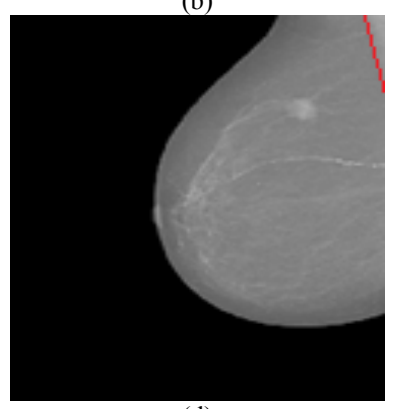

(d)

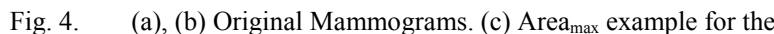
pectoral muscle region. (d) Area $a_{\min }$ example for the pectoral muscle region.

When the SSEM method is used, the pixel at the border of the candidate pectoral muscle is marked as 255 . Thus, the resulting image of SSEM is converted a binary image with a threshold value of 0.99 (Figure 5(b)) and the pectoral muscle border is strengthened by morphological operators (Figure $5(c))$. The image, which has been converted into a binary image with enhanced pectoral muscle strength, is cleaned from images smaller than the Area $a_{\text {min. }}$ The cleaned image is added with the previously obtained rough breast region. Then to create the pectoral muscle area, a line is drawn starting from the upper left corner and covering the pectoral muscle corner from top to bottom and left to right (Figure 5(c)). The area between these lines and the border of the candidate pectoral muscle region is filled. Two regions with the largest area are selected to detect the pectoral muscle region (Figure 5(d)). The region located at the upper left of the other region and is bigger than the Area $a_{\min }$ and smaller than Area $a_{\max }$ is selected as the pectoral muscle region (Figure 5(e)). There may be residual defects at the border of the candidate pectoral muscle region, sometimes after morphological processing (Figure 6(b)). Similar to the SSEM method, while obtaining the boundaries of the pectoral muscle region to get rid of these disorders, the pectoral muscle location information was taken at an angle of about $30^{\circ}-45^{\circ}$ from the top down and left to right, and the muscle border was created. Finally, a linear interpolation method was used to complete the missing points on the obtained boundaries, and the result obtained was regarded as the final muscle border (Figure 6(c)).

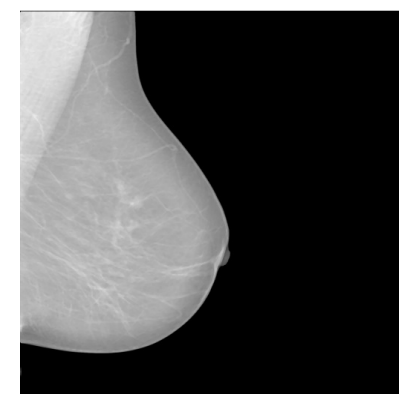

(a)

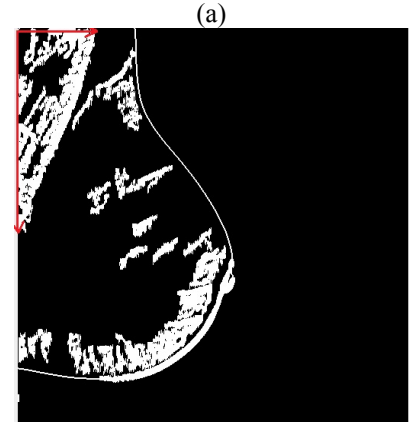

(c)

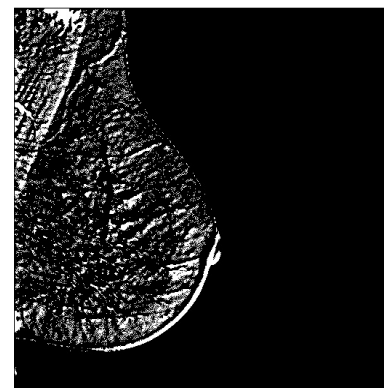

(b)

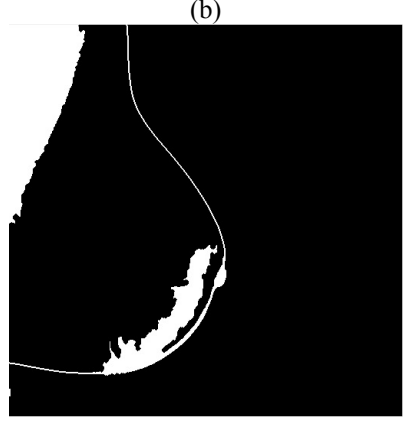

(d)



(e)

Fig. 5. (a) The original mammogram. (b) The binary image of the SSEM result. (c) The mammogram after morphological processing. (d) Mammogram with the selected two largest areas. (e) Mammogram with the rough pectoral muscle area determined.

The final muscle border obtained for a sample mammogram and the muscle margin plotted using ground truth values given by the experts for the same mammogram are presented in Figure7. The flow chart of all the procedures used for boundary detection and for determining the candidate pectoral muscle region is presented in Figure 8.

\section{EXPERIMENTAL STUDIES}

The proposed algorithm has been tested on 60 mammograms received from the INbreast database. The obtained results were evaluated in terms of false positive rate (FPR), false negative rate (FNR) and sensitivity with groundtruth data given by the radiologists. False positive (FP) is the 
pixel found by the algorithm as the region of the pectoral muscle but detected as a different tissue by the radiologist, false negative (FN) is the pixel shown by the radiologist as pectoral muscle but detected as a different tissue by the algorithm and true positive (TP) is the pixel determined by the radiologist as the pectoral muscle and is also found as the region of the pectoral muscle by the algorithm. FPR, TPR and sensitivity are defined by (2)-(4) and are presented in Table I.

$$
\begin{aligned}
& \mathrm{FPR}=\mathrm{FP} /(\mathrm{TP}+\mathrm{FP}) \\
& \mathrm{TPR}=\mathrm{TP} /(\mathrm{TP}+\mathrm{FN}), \mathrm{FNR}=1-\mathrm{TPR}
\end{aligned}
$$$$
\text { Sensitivity }=\mathrm{TP} /(\mathrm{TP}+\mathrm{FN})
$$

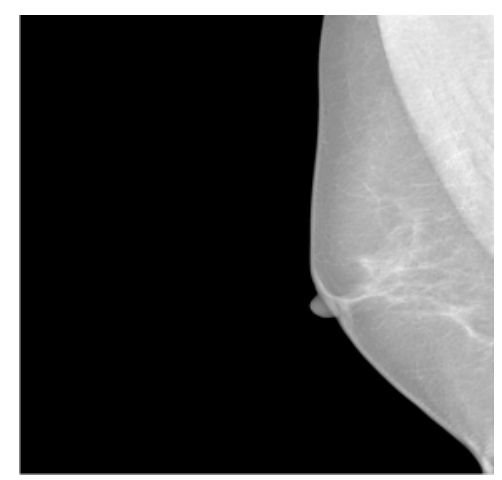

(a)

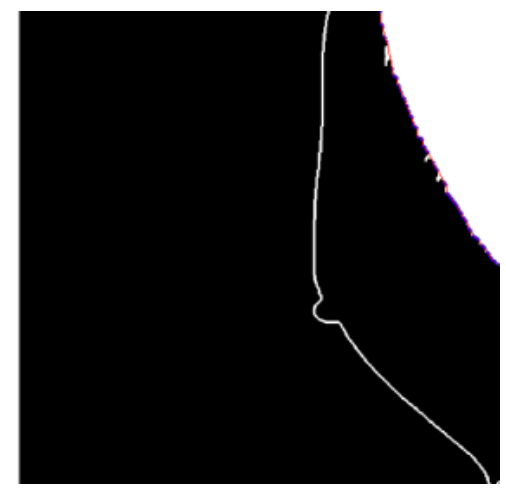

(b)

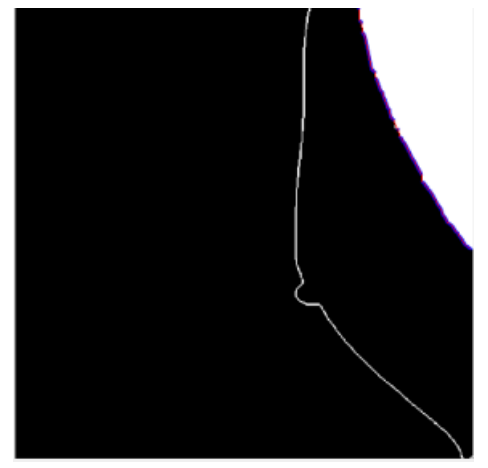

(c)

Fig. 6. (a) Original mammogram. (b) Obtained mammogram image of rough pectoral muscle boundaries. (c) Mammogram image of the final borders obtained by linear interpolation.

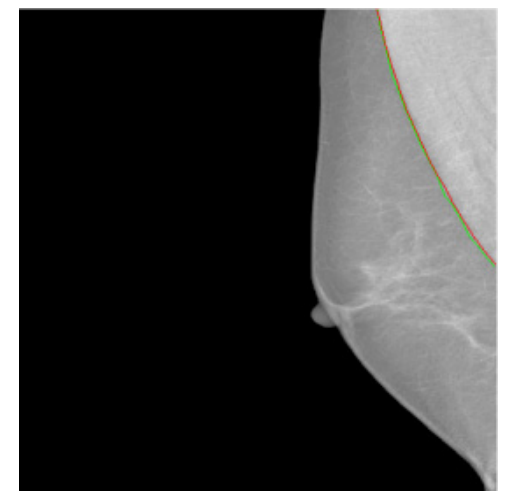

Fig. 7. Muscle limit (red) plotted using ground truth values given by experts on a sample mammogram and muscle limit (green) found by the proposed method.

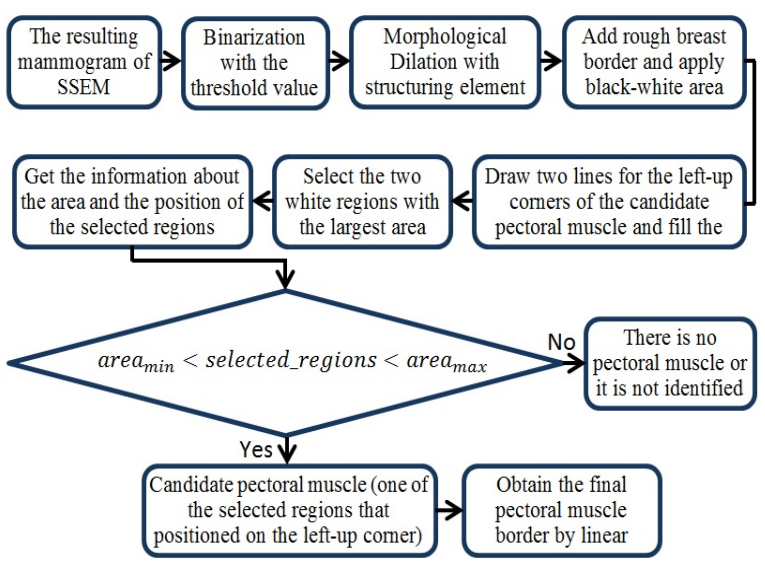

Fig. 8. Flow chart of candidate pectoral muscle region and border determination

As shown in Table I, the proposed method was able to detect the pectoral muscle region with an average sensitivity of $95.6 \%$ for the 60 mammogram images. Also, in order to show the performance of the proposed method on different mammogram images, images of pectoral muscle boundary detection applications for four different mammogram images are presented in Figure 9.

TABLE I. PERFORMANCE MEASURES

\begin{tabular}{|c|c|c|c|}
\hline $\begin{array}{c}\text { Performance } \\
\text { Measures } \\
\text { Parameters }\end{array}$ & $\begin{array}{c}\text { Average FP } \\
\text { Rate }\end{array}$ & $\begin{array}{c}\text { Average FN } \\
\text { Rate }\end{array}$ & $\begin{array}{c}\text { Average } \\
\text { Sensitivity }\end{array}$ \\
\hline Results & 0,0274 & 0,04331 & 0,9566 \\
\hline
\end{tabular}

\section{CONCLUSIONS}

In this study, a new method has been proposed to determine the pectoral muscle and its borders on digital mammograms. In this method, the pectoral muscle border was determined using a technique based on geometric features of the pectoral muscle and neighborhood relations. The boundaries for the rough pectoral muscle region have been strengthened and adjusted and the linear interpolation method has been used to complete 
the missing points within this boundary and to establish border continuity. The recommended method was tested using 60 mammograms from the INBreast database. According to the results obtained, it has been shown that the proposed method can detect the pectoral muscle margin with an average of $95.6 \%$ sensitivity



(a)

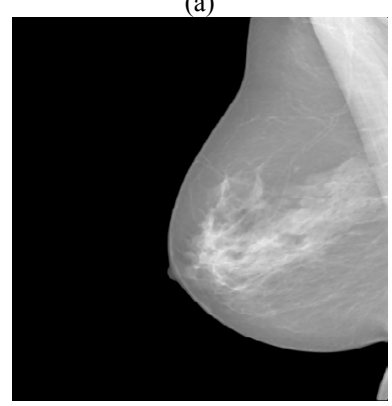

(c)

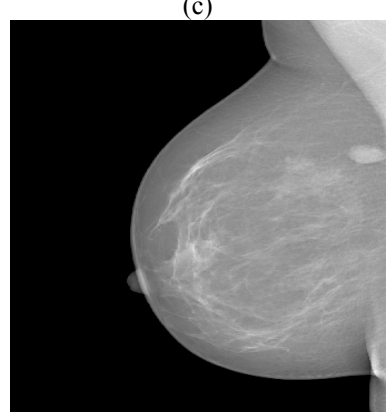

(e)

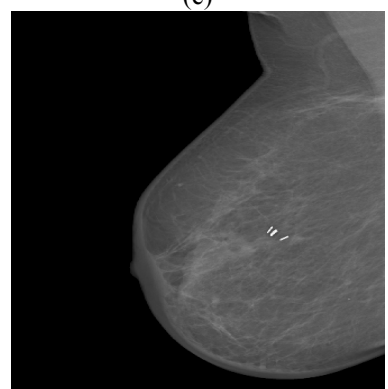

(g)

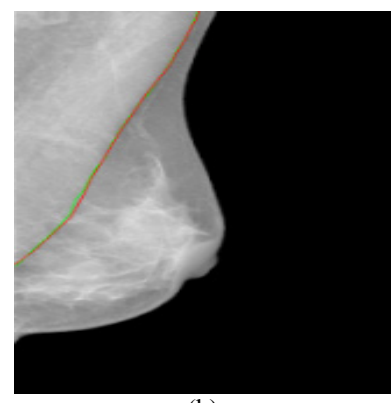

(b)

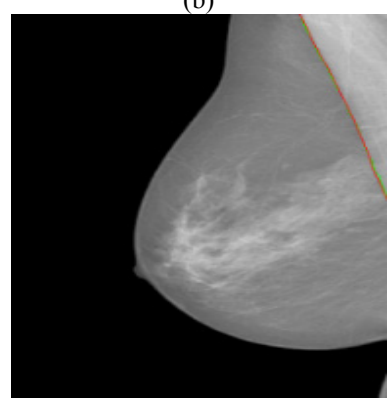

(d)

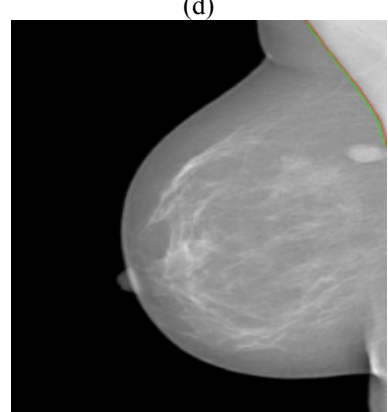

(f)

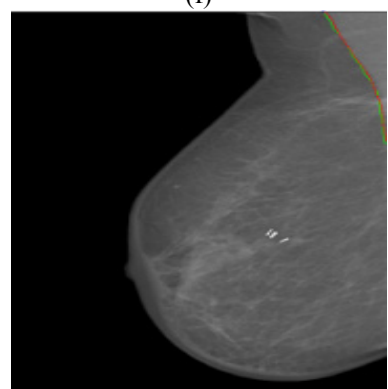

(h)
Figure 9. (a, c, e, g) Original Mammograms. (b, d, f, h) Muscle boundaries (red) drawn using ground truth values given by experts and muscle boundaries (green) found by the proposed method.

\section{ACKNOWLEDGEMENT}

The authors would like to thank the "Breast Research Group, INESC Porto, Portugal" for allowing the use of the INbreast database of mammograms used in this study.

\section{CONFLICTS OF INTEREST}

The authors declare that there is no conflict of interest regarding the publication of this article.

\section{REFERENCES}

[1] S. Radhika, S. Sasikala, R. Mariappan, "Three-dimensional reconstruction of microcalcification clusters from CC and MLO views", 2nd International Conference on Electronics and Communication Systems, pp. 607-611, 2015

[2] M. Tan, B. Zheng, "Development of a new case based computer-aided detection scheme for screening mammography", IEEE EMBS Conference on Biomedical Engineering and Sciences, pp. 24-29, 2016

[3] I. Christoyianni, A. Koutras, E. Dermatas, G. Kokkinakis, "Computer aided diagnosis of breast cancer in digitized mammograms", Computerized Medical Imaging and Graphics, Vol. 26, No. 5, pp. 309319,2002

[4] K. Doi, H. K. Huang, "Computer-aided diagnosis (CAD) and imageguided decision support", Computerized Medical Imaging and Graphics, Vol. 31, No. 4-5, pp. 195-197, 2007

[5] R. J. Ferrari, R. M. Rangayyan, J. E. L. Desautels, R. A. Borges, A. F. Frere, "Automatic Identification of the Pectoral Muscle in Mammograms", IEEE Transactions on Medical Imaging, Vol. 23, No. 2 , pp. 232-245, 2004

[6] A. Sultana, M. Ciuc, R. Strungaru, "Detection of pectoral muscle in mammograms using a mean-shift segmentation approach", 8th International Conference on Communications, pp. 165-168, 2010

[7] S. M. Kwok, R. Chandrasekhar, Y. Attikiouzel, "Automatic pectoral muscle segmentation on mammograms by straight line estimation and cliff detection", The Seventh Australian and New Zealand Intelligent Information Systems Conference, pp. 67-72, 2001

[8] M. Yam, M. Brady, R. Highnam, C. Behrenbruch, R. English, Y.Kita, "Three-dimensional reconstruction of microcalcification clusters from two mammographic views", IEEE Transactions on Medical Imaging, Vol. 20, No. 6, pp. 479-489, 2001

[9] X. Weidong, L. Lihua, L. A Wei, "Novel pectoral muscle segmentation algorithm based on polyline fitting and elastic thread approaching", 1st International Conference on Bioinformatics and Biomedical Engineering, pp. 837-840, 2007

[10] P. Miller, S. Astley, "Classification of breast tissue by texture analysis", Image and Vision Computing, Vol. 10, No. 5, pp. 277-282, 1992

[11] A. Khademi, S. Farhang, A. Venetsanopoulos, K. Sridhar, "Region, Lesion and Border-Based Multiresolution Analysis of Mammogram Lesions Lecture Notes in Computer Science", International Conference Image Analysis and Recognition, pp. 802-813, 2009

[12] K. Ganesan, U. Rajendra Acharya, K. Chua Chua, L. Choo Min, K. T. Abraham, "Pectoral muscle segmentation: A review", Computer Methods and Programs in Biomedicine, Vol. 110, No. 1, pp. 48-57, 2013

[13] M. Lee, Y. Chen, S. Kim, J. Kim, "Segmentation of the Pectoral Muscle Boundary in Breast MR Images", IEEE 11th International Conference on Bioinformatics and Bioengineering, pp. 323-326, 2011

[14] C. C. Liu, C. Y. Tsai, J. Liu, C.-Y. Yu, S. S. Yu, "A pectoral muscle segmentation algorithm for digital mammograms using Otsu thresholding and multiple regression analysis", Computers \& Mathematics with Applications, Vol. 64, No. 5, pp. 1100-1107, 2012

[15] S. S. Yu, C. Y. Tsai, C. C. Liu, "A breast region extraction scheme for digital mammograms using gradient vector flow Snake", 4th International Conference on New Trends in Information Science and Service Science, pp. 715-720, 2010

[16] S. Sreedevi, E. Sherly, "A Novel Approach for Removal of Pectoral Muscles in Digital Mammogram", Procedia Computer Science, Vol. 46 pp. 1724-1731, 2015

[17] I. C. Moreira, I. Amaral, I. Domingues, A. Cardoso, M. J. Cardoso, J. S. Cardoso, "INbreast: Toward A Full-Field Digital Mammographic Database", Academic Radiology, Vol. 19, No. 2, pp. 236-248, 2012 
[18] J. L. Starck, F. Murtagh, E. J. Candes, D. L. Donoho, "Gray and color image contrast enhancement by the curvelet transform", IEEE Transactions on Image Processing, Vol. 12, No. 6, pp. 706-717, 2003

[19] J. S. Lee, "Digital Image Enhancement and Noise Filtering by Use of Local Statistics", IEEE Transactions on Pattern Analysis and Machine Intelligence, Vol. PAMI-2, No. 2, pp. 165-168, 1980

[20] E. S. Yelmanova, Y. M. Romanyshyn, "Medical image contrast enhancement based on histogram", IEEE 37th International Conference on Electronics and Nanotechnology, pp. 273-278, 2017

[21] M. A. Abeed, A. K. Biswas, M. M. Al-Rashid, J. Atulasimha, S. Bandyopadhyay, "Image Processing With Dipole-Coupled Nanomagnets: Noise Suppression and Edge Enhancement Detection", IEEE Transactions on Electron Devices, Vol. 64, No. 5, pp. 2417-2424, 2017

[22] M. Regodic, L. Gigovic, Z. Bajic, S. Vasiljević "Contrast Enhancement of Colour Digital Images", Tehnicki vjesnik, Vol. 24 No. 3, pp. 935-941, 2017

[23] S. H. Wang, T. M. Zhan, Y. Chen, Y. Zhang, M. Yang, H. M. Lu, H. N. Wang, B. Liu, P. Phillips, "Multiple Sclerosis Detection Based on Biorthogonal Wavelet Transform, RBF Kernel Principal Component Analysis, and Logistic Regression", IEEE Access, Vol. 4, pp. 75677576, 2016

[24] M. Vetterli, J. Kovacevic, Wavelets and Subband Coding, Prentice Hall PTR, 1995 